\title{
Processus dynamique, pratiques hybrides et engagement de la recherche : les médiations culturelles en débat
}

Dynamic process, hybrid practices and the involvement of research: discussing cultural mediation

Michèle Gellereau

\section{CpenEdition}

Journals

Édition électronique

URL : http://journals.openedition.org/edc/7493

DOI : 10.4000/edc.7493

ISSN : 2101-0366

Éditeur

Université de Lille

Édition imprimée

Date de publication : 1 juin 2018

Pagination : 57-74

ISBN : 978-2-917562-19-2

ISSN : $1270-6841$

Référence électronique

Michèle Gellereau, «Processus dynamique, pratiques hybrides et engagement de la recherche : les médiations culturelles en débat », Études de communication [En ligne], 50 | 2018, mis en ligne le 01 juin 2020, consulté le 02 janvier 2020. URL : http://journals.openedition.org/edc/7493 ; DOI : 10.4000/edc. 7493 
Processus dynamique, pratiques hybrides et engagement de la recherche : les médiations culturelles en débat

Dynamic process, hybrid practices and the involvement of research: discussing cultural mediation 
Résumé / Abstract

L'article expose la manière dont l'évolution des pratiques de médiation culturelle, les débats sur la notion ont été saisis par Études de communication dans des approches différentes mais toujours fondée sur des expériences de recherche, de formation, de pratiques professionnelles ou amateures. Après un bref retour sur les recherches en $\mathrm{SIC}$, trois questions sont considérées pour en saisir les enjeux : la question des limites de la définition de la médiation comme un "entredeux "; celle du caractère hybride des médiations, celle des modalités de la recherche en médiation culturelle. La notion, toujours en débat, est analysée comme processus dynamique de transformation, de rencontre, s'actualisant au fil des développements technologiques dans des pratiques hybrides.

Mots-clés : médiation culturelle, Études de communication, entre-deux, hybridité.
This article considers how the journal Etudes de communication has taken up the notion of cultural mediation and the evolution of cultural mediation practices. Our analysis shows that despite different approaches, the question of cultural mediation is consistently addressed on the basis of research experiences and professional or "amateur" practices. After giving an account of research conducted on cultural mediation within the field of Information and communication science, we discuss three points: the limits of considering mediation as a "between space"; the hybrid nature of mediation devices; and research methods in the area of cultural mediation. The notion of cultural mediation, still under debate, is analyzed as a dynamic process of transformation and encounter, evolving continually in relation to technological developments and changing hybrid practices.

Keywords: cultural mediation, Etudes de communication, between space, hybridity. 


\section{Introduction}

Quand paraît en 1998 le n² 21 d’Études de communication (EDC) « Médiations culturelles : dispositifs et pratiques », cela fait déjà quelques années que la réflexion universitaire en sciences de l'information et de la communication et en sociologie sur la question connaît un certain développement (Hennion, 1990 ; Caune, 1995 ; Caillet et Lehalle, 1995), que des formations à la « médiation culturelle et communication » existent à l'université, notamment à Lille ; un groupe «Culture et médiation » vient de se créer au sein de la SFSIC (1996) qui marque entre autres l'entrée de collègues en muséologie au sein des Sciences de l'Information et de la communication (SIC); un des moments de cette étape sera l'organisation de Journées d'études en mars 1999 à Lille qui ont donné lieu à un ouvrage, Médiation des cultures (Gellereau et al., 2000). Ces deux publications de GERICO ${ }^{1}$ mobilisent des collègues de Lille et d'autres universités qui participent activement au développement des travaux et formations en SIC sur la médiation dans une période où les ouvrages se multiplient sur le sujet (notamment : Caune, 1999 ; Lamizet, 2000). À cette époque et dans les quelques années qui suivent, les travaux tentent de fonder ou d'interroger la notion de " médiation culturelle » qu'il faut construire, affirmer et défendre dans certains cas, au moins expliciter.

Quand paraissent 17 ans et 18 ans plus tard, les numéros 45 et 46 d'EDC "Les médiations des patrimoines vers la culture numérique? » dont plusieurs articles utilisent la notion de médiation culturelle, totalement intégrée aux discours, on en interroge désormais les liens avec la nouvelle médiation à questionner, celle du numérique. Généralisation d'emploi, banalisation, institutionnalisation de la notion de médiation culturelle ? De fait, entre ces deux moments, les pratiques institutionnelles de la médiation culturelle, les enseignements et les recherches se sont développés et structurés ; les étudiants ont trouvé des emplois dans les lieux et institutions qui ont professionnalisé les activités de médiation culturelle, les travaux sur la notion (mémoires, thèses, ouvrages, articles, colloques) ont proliféré, notamment en sciences de l'information et de la communication et en sociologie, se sont internationalisés (Lafortune, 2012). Entre 1998 et 2016, la revue Études de communication a pour sa part continué à publier des articles sur la question ou mobilisant la notion tout en s'intéressant aux liens de celle-ci avec d'autres concepts (celui d'interprétation dans le dossier du $\mathrm{n}^{\circ} 24$ de 2001) ou d'autres questionnements (dossier sur les espaces urbains par exemple dans le $n^{\circ} 31$ de 2008). Puis, la question de la médiation numérique s'est trouvée au centre de nombreux travaux en SIC et une recontextualisation de la notion de médiation culturelle s'est opérée.

Cet article n'a pas la prétention de refaire un panorama de l'évolution de la médiation et des recherches sur le sujet, ni de proposer une analyse 
exhaustive des articles publiés. En tant qu'auteure coordinatrice ou publiante dans les numéros considérés, il me semble difficile de saisir la revue comme un corpus à analyser de l'extérieur. II s'agira plutôt de mettre en perspective la manière dont l'évolution des pratiques de médiation culturelle, les débats sur la notion ont été saisis par Études de communication, au-delà des différences d'analyse, dans une approche spécifique toujours fondée sur des expériences de recherche, de formation, de pratiques professionnelles ou amateures et de comprendre comment les recherches ont évolué dans cette attention constante aux pratiques de médiation. Ce point de vue sera spécifié à partir de la mise en discussion de quelques questions qui animent certains articles d'Études de communication et restent en débat.

Ma contribution se fera en quatre temps. Le premier rappellera brièvement la manière dont la question de la médiation culturelle est saisie dans les travaux de recherche en essayant de pointer quelques constantes et différences que les contributeurs d'Études de communication mettent également en lumière. Trois questions seront ensuite considérées, en essayant d'en saisir quelques enjeux : la question des limites de la définition de la médiation comme un " entre-deux »; celle du caractère hybride des médiations, celle des modalités de la recherche en médiation culturelle. Ces trois entrées ont comme point commun de considérer qu'il n'y a pas de médiation en soi, mais des médiations ancrées dans des pratiques et d'interroger les évolutions de la notion.

\section{1. \\ La médiation culturelle au cœur des pratiques artistiques et patrimoniales}

Dans un article publié récemment dans un ouvrage collectif sur la médiation (Servais et al., 2016) intitulé « Médiations culturelles et patrimoniales : partager des expériences culturelles pour construire du commun » (Gellereau, 2016), je suis revenue sur diverses constantes ou discussions en SIC que je ne commenterai pas ici. Dans le bref développement qui suit, il s'agira de préciser que cette notion n'est en rien un concept figé, mais qu'elle évolue et est discutée dans un creuset d'enjeux culturels et sociaux.

De manière récurrente, dans la littérature de la fin des années 90 , la notion de médiation culturelle est opposée à l'idée d'évidence du sens des œuvres (Caune, 1999). Elle désigne des actions d'accompagnement, des langages et des dispositifs d'interprétation des œuvres permettant aux publics de s'approprier les objets culturels (Lamizet, 2000). Elle s'intéresse à la manière dont les pratiques culturelles font sens et au rôle des publics dans un processus de démocratisation culturelle en remettant en cause un modèle pédagogique d'instruction (Caillet et Lehalle, 1995) dans l'objectif, par du lien, de réparer une «fracture » entre œuvres et publics (Caune, 1999). Ces points sont largement 
développés et discutés dans plusieurs articles du n² 21 de la revue (1998). Le débat sur le rôle politique de la médiation culturelle permet de mettre en évidence comment la médiation peut devenir prescriptive (Da Lage, 1998) ou comment, en considérant la médiation comme un phénomène complexe de partages de cultures, légitimes ou « illégitimes » on crée des manières d'interpréter ou de raconter des expériences sociales et culturelles (Gellereau, 1998, Giet, 1998).

Les intérêts de la recherche ont par ailleurs évolué au fil du temps et du développement des activités de médiation dans les milieux professionnels. $\mathrm{Au}$ début des années 2000, de nombreux chercheurs publient des analyses de cas concrets de médiation et on constate un engouement important des institutions culturelles pour la médiation, dans l'objectif de transmettre des savoirs ou de conquérir des publics. Bordeaux (2011), ou Chaumier et Mairesse (2013) font des synthèses intéressantes de cette période riche en expérimentations et travaux sur la médiation telle que pratiquée dans les institutions culturelles. Bordeaux et Caillet (2013) montrent aussi comment se développent le dialogue entre disciplines et l'engagement des modèles théoriques de l'information-communication, ainsi que le rôle des chercheurs et de la revue Cultures et musées dans l'accompagnement scientifique des évolutions.

D'autres auteurs (Hennion, 2004a) s'intéressent à la place accordée au rôle des publics et des amateurs ou passionnés comme co-producteurs d'interprétations et d'une médiation considérée comme outil de partage et de

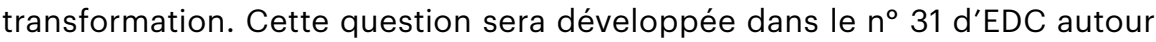
des pratiques patrimoniales des espaces urbains tout comme dans plusieurs articles analysant des pratiques créatives dans l'appropriation des œuvres dans les $n^{\circ}$ 45-46. Études de communication a en effet apporté une contribution notable à la question des amateurs et questionné dans ces deux périodes la question du rapport médiation professionnelle/médiation amateure (Leclercq, 1998 ; Van Braekel, 1998 ; Da Lage, Gellereau, Laudati, 2008 ; Chupin 2016). Dans ce mouvement, la revue est entrée pleinement dans les débats sur le rôle de la médiation dans la reconnaissance de la diversité des usages culturels et des partages de savoirs et expériences entre professionnels, amateurs et publics. Ce phénomène correspond d'ailleurs à l'engagement d'une partie des acteurs culturels, notamment des musées et du patrimoine dans « le souci du public » (Le Marec, 2007, 7).

À partir des années 2000, le développement du numérique et des pratiques du web s'empare de la médiation et repose la question du rôle de la médiation dans le partage des cultures. "Par ailleurs, le développement important des médiations numériques dans le domaine de la culture a, entre autres choses, produit un rapprochement du monde des musées avec le monde des bibliothèques ou du multimédia, et a renforcé la réflexion sur les médiations techniques permettant aux publics d'intervenir dans les dispositifs de médiation » (Gellereau, 2016, 110). Les travaux sur les médiations numériques dans l'espace public, en musée ou dans les institutions culturelles enrichissent les 
réflexions sur la participation des publics et sur l'inventivité ou les innovations créées par les médiations comme le montrent plusieurs articles d'EDC 45 et 46. La médiation n'apparaît plus seulement comme un lieu intermédiaire mais comme un ferment de transformation qui engage acteurs de la médiation et publics dans l'interprétation et aussi la transformation des objets culturels, ce sera l'objet des points suivants de cet article.

Du point de vue méthodologique, on peut constater que la notion de médiation est travaillée dans ces numéros de la revue dans la perspective d'analyse de pratiques de médiation et de questionnement de " ce qui est en train de se faire » (Tardy et Renaud, 2015, 11) ; les contributeurs s'appuient sur des auteurs de référence qui pour certains tentent de construire la médiation en concept mais ils ne visent pas à formaliser le concept pour lui donner " un " sens ou " une " définition modélisante. Il s'agit plutôt, dans le $n^{\circ} 21$, non de définir la médiation mais de penser des pratiques culturelles en terme de médiation (Gellereau, 1998a, 8). Dans les n 45 (2015) et 46 (2016), il s'agit d'analyser les enjeux des médiations patrimoniales « ressaisies par la culture numérique " (Tardy et Renaud, 2015, 13) en analysant des situations ou dispositifs concrets. Les réflexions menées par les différents auteurs sont donc toujours ancrées dans la diversité des pratiques de médiation et comme le souligne Pierre Delcambre, le « terme de médiation ne vaudrait dès lors qu'au pluriel : de multiples acteurs opèrent d'incessantes médiations " (Delcambre, $1998,143)$. Ces questionnements traversent un grand nombre de travaux en SIC entre 1995 et 2017, alors que d'autres travaux se focalisent sur les définitions du concept, ou sur les techniques de médiation et ont parfois une visée modélisatrice pour proposer de bonnes pratiques, ce qui n'est pas l'axe retenu par la revue. Celle-ci a en effet le souci de faire émerger et dialoguer des travaux dont les approches sont différentes mais qui toutes interrogent les processus de médiation dans leur capacité à produire des dynamiques de construction du sens, de rencontre et de partage d'interprétations. Dans ce même esprit, certaines questions me semblent au cœur des évolutions et de l'enrichissement continu de la notion.

\section{2.}

\section{«Entre-deux », rencontre et monde commun. Comment penser la dynamique de la médiation?}

La première question est celle que l'on peut rapidement caractériser comme une définition statique de la médiation à partir de l'image de "l'entredeux ». En effet, les images de l'entre-deux, des " espaces » intermédiaires, sont fréquemment reprises pour décrire la médiation. Dans le Dictionnaire de la muséologie, Desvallées-Mairesse $(2011,215)$ estiment que « la médiation se place dans un entre-deux, dans un espace qu'elle cherchera à réduire en provoquant un rapprochement, voire une relation d'appropriation ». Différents auteurs 
décrivent ainsi le fait que la médiation invite à penser ce qui se joue entre les œuvres et le public ou pour insister sur le fait que la médiation se retrouverait dans un espace "médian ", voire neutre, entre art et public. Ayant employé également cette image au début de mes recherches, je suis ensuite revenue sur la question de la séparation des mondes qu'elle suggère : « je proposerai de considérer que la médiation n'est pas neutre, ni un entre-deux détaché des mondes qu'elle prétendrait relier » (Gellereau, 2016). Pourtant, si on retrouve dans l'« entre-deux » une tentative de produire une définition permettant de se représenter une médiation souvent difficile à saisir (Servais 2016), l'image semble très peu mobilisée dans les analyses concrètes des pratiques proposées dans la revue. Comment interpréter alors cette quasi-absence?

Est-ce parce qu'elle donne une vision statique d'une relation qui est en fait un processus, une dynamique de partage ? Un propos de Laplantine et Nouss sur la question du métissage appuie cette hypothèse. Ces auteurs en effet considèrent celui-ci comme une pensée de la transformation :

"Si la pensée du métissage est bien une pensée de la médiation qui se joue dans les intermédiaires, les intervalles et les interstices à partir de croisements et d'échanges, elle ne saurait se réduire à l'entre et à l'entre-deux qui sont des catégories spatiales. C'est [...] une pensée de la tension, c'est-à-dire une pensée résolument temporelle [...] une pensée de la multiplicité née de la rencontre »(Laplantine et Nouss, 1997, 83).

Cette vision que je proposerai volontiers de rapporter à la médiation culturelle me semble plus riche que la simple vision spatiale car elle décrit un processus de transformation (Hennion, 2003) et correspond aux phénomènes complexes de médiation observés dans différents articles de la revue qui ne peuvent se résumer à une place " entre » œuvre et public. Par ailleurs, sur le plan de l'analyse des pratiques, Marie-Christine Bordeaux alerte en 2007 sur les problèmes que peut poser l'analyse de la médiation en termes d'entre-deux pour le spectacle vivant :

«Cette vision, qui place le médiateur comme agent de l'entre-deux d'une communication problématique entre des objets et des visiteurs, rend mal compte de la complexité de l'expérience esthétique et culturelle : la situation de médiation est un tissu serré de pactes de réception, de dispositifs de communication et de transmission, d'élaboration croisées » (Bordeaux, 2007, 10) ; " elle agit comme un espace de production d'objets hybrides : objets 'impurs', 'situations intermédiaires', selon Goffman ; 'manifestations indirectes', dirait Genette » (Bordeaux 2007, 11).

Plusieurs textes d'EDC 21 traitent du spectacle vivant, ou de dispositifs médiatiques décrivant d'ailleurs la médiation non pas comme se situant « entre » deux pôles mais comme une dynamique, un élément même de l'action collective qui produit l'art (Leclercq 1998, 26 ; Van Braekel 1998, 31, 32, 35, 41). Pour E. Leclercq qui mobilise les travaux d'Howard Becker, "les contenus comme 
les usages d'une œuvre dépendent en dernière analyse d'un jeu complexe de collaborations " (Leclercq, 1998, 13), rappelons que pour Becker les différents acteurs et les publics des chaînes de coopération sont intégrés au monde de l'art ; Leclercq propose une analyse des collaborations et réseaux dans le spectacle vivant où « la fracture ne désigne plus la séparation entre deux mondes mais entre les moments où l'œuvre existe et celles où elle n'est envisagée que comme projet (ou un souvenir) » (Leclercq, 1998, 14). D'autres auteurs d'EDC 21 montrent que la médiation est davantage un processus d'« interpénétration ", et de production d'une nouvelle réalité (Da Lage 1998, 70) ou un processus de rencontre (Gellereau, 1998b). Dans ces cas, la médiation apparait davantage comme un élément dynamique qui produit des transformations que comme un espace entre œuvre et public. On retrouve cette même idée de transformation par la co-construction dans plusieurs travaux sur le patrimoine analysés dans les $n^{\circ} 45$ et $46 d^{\prime} E D C$. Centrés sur la médiation patrimoniale et numérique, la médiation est elle-même une co-production, une expression dialogale : "la médiation provient d'un dialogue engagé entre l'édifice existant avec la projection en mapping » (Georgescu-Paquin, 2015, 68), expressions artistiques et médiation patrimoniale se complètent dans une co-construction du sens (Bonaccorsi et Croissant, 2015). La question de l'articulation d'un ensemble d'éléments (formes langagières, communicationnelles, technologiques, processus relationnels...) dans un dispositif singulier de médiation est centrale dans différentes analyses, ce qui avait déjà été développé par Davallon (2004).

La notion « d'entre-deux » ne me parait pas dans ce cas heuristique pour décrire une rencontre produite par une activité de médiation qui ne se situe pas entre deux mondes statiques mais s'ancre dans la dynamique des pratiques des acteurs et des publics qui se retrouvent dans un monde partagé qui évolue. Si elle permet de prendre en compte un espace de négociation, elle peine cependant à rendre compte d'une part du caractère circulaire d'une médiation qui pose la question relevée par Sylvette Giet citant Hennion : " comment faisons-nous le monde qui nous fait ? " (Giet, 1998, 120) et d'autre part l'actualisation d'un dialogue dynamique qui se construit entre différents acteurs ou entités dont le public fait partie. Comme le souligne J. Le Marec dans la postface du $n^{\circ} 24$ d'EDC sur l'interprétation :

"Certaines approches amènent très concrètement à penser la circulation des représentations sur un autre mode que celui de la séparation entre des univers sociaux différents, comme par exemple, les pôles de la production et de la réception ou bien alors, les pôles du savoir savant et du savoir de sens commun. Par exemple, dans le cas du milieu muséal, les concepteurs d'exposition, les visiteurs et les évaluateurs partagent une culture commune » (Le Marec, 2001, 127).

Décrites dans les articles de la revue comme projets qui se tendent dans le temps, ferments de transformation, processus de rencontre, les médiations culturelles développent des formes de partage non réductibles à un « entre- 
deux ». Cette vision dynamique de la médiation est renforcée par deux autres questionnements qui apparaissent à la lecture des articles de la revue : la question de l'hybridation déjà soulevée dans cette partie sur l'entre-deux (Bordeaux 2007) et celle de la participation des différents acteurs dans cette dynamique.

\section{3. \\ Hybridité, hybridation et création numérique. Quelles évolutions des médiations?}

Très présente dans les analyses des médiations numériques, amplement développée ces dernières années, la question de l'hybridité n'est cependant pas nouvelle et s'intéresse aux objets-médiateurs ou objets de médiation. Plusieurs articles d'EDC 21 posent la question de l'hybridité de la médiation. D. Vandiedonck nous rappelle que "Dans la tradition issue des travaux de A. Hennion, l'étude des médias s'intéresse à des objets hybrides qui 'font' médiation et le secteur de la musique est balisé par de tels objets : boîtiers de disques, plaquettes et programmes des concerts [...] »(Vandiedonck 1998, 49). Pierre Delcambre reprend dans sa post-face d'EDC 21 cette question de l'hybride et fait remarquer que " saisir les objets culturels sous l'angle de la médiation évite une sémiologie trop réductrice en intégrant l'analyse du sens de ces objets médiateurs pour l'ensemble des acteurs : on s'intéresse dès lors aux pratiques complexes dont la production est l'occasion » (Delcambre 1998, 139).

À l'aune du numérique, la question semble relever d'une évolution dans la manière de saisir la médiation culturelle en la reliant notamment à la question de la création et de l'interactivité. Plusieurs articles d'EDC 45 et 46 relèvent en effet la question de l'hybridation des formes dans les musées ou la ville, à propos de vidéo (German, 2016, 68) de mapping (Georgescu-Paquin, 2015), ou de dispositifs « interactifs " (Dalbavie et al., 2016 ; Lambert et al., 2015). SeIon ces auteurs, le processus de médiation crée des objets nouveaux, parfois éphémères, qui sont à la fois l'œuvre et la médiation, ou une transformation de l'œuvre ou du patrimoine dans la médiation. À propos d'itinéraires numériques et artistiques à travers le patrimoine urbain, on peut constater que " ces productions mettent en œuvre une médiation culturelle à la fois de et par l'art » (Lambert et al., 2015, 122). Les mêmes auteurs estiment que "I'hybridation de formes et des médias liés au numérique [...] participent à construire des médiations renouvelées... » (Lambert et al., 2015,126). Pour A. Georgescu-Paquin les mapping-video sur le patrimoine bâti produisent une hybridation entre $œ u v r e$ et dispositif de médiation qui n'est pas pensée ici comme une médiation, mais comme une transformation favorisant la médiation et une nouvelle interprétation du bâtiment » (Georgescu-Paquin, 2015, 55). 
Dans ces exemples, on voit comment cette question de l'hybridation peut être reliée au questionnement sur l'entre-deux ; la médiation n'est pas ici un discours d'accompagnement entre l'œuvre et le public, elle peut être un hybride, à la fois nouvelle œuvre interprétative, expérience esthétique, projet culturel. Elle prend forme dans des langages, des écritures qui proposent une appropriation de l'œuvre ou du patrimoine fondée sur une nouvelle expérience esthétique de celle-ci. C'est le cas du projet le Vase qui parle décrit par Dalbavie, Da Lage et Gellereau dans EDC 46 où l'objet de médiation devient un quasi-objet d'art lui-même, non pas ajouté à l'oeuvre originale mais réalisé pour en proposer une pratique nouvelle, centré sur la réalisation d'une expérience inhabituelle pour le visiteur qui comme les autres cas cités précédemment engagent le corps du public.

Car la question de l'hybridité ne vaut pas simplement pour l'objet-médiateur ou le dispositif, elle se pose aussi dans la manière de construire une relation à l'objet. Les expériences décrites mettent en évidence le fait que c'est dans la pratique des publics que s'actualisent des formes d'interprétation et de médiation sensible qui transforment l'objet ou le monument. Dans la perspective de comprendre ce qu'est la médiation, C. Servais estime que " c'est la fiction qui permet de relier la technique au toucher, c'est-à-dire la manière dont les œuvres ou les dispositifs nous 'font' quelque chose » (Servais, 2016, 133). Selon elle, la fiction « n'est pas la création d'un monde imaginaire opposé au monde réel. Elle est le travail qui opère des dissensus [...] en construisant des rapports nouveaux entre l'apparence et la réalité, le singulier et le commun, le visible et la signification " (Servais, 2016, 154). II me semble que cette manière de considérer la médiation-fiction invite à penser davantage la question de l'hybridation au regard des relations et pratiques des publics, ce qui peut enrichir cette question de l'hybridation. Ainsi, « on ne peut penser la question du numérique séparément de celle des nouvelles pratiques que génèrent, non la technologie numérique en soi, mais son hybridation avec des pratiques culturelles, esthétiques, sociales »(Dalbavie et al., 2016, 124).

Ainsi, au-delà de la question de l'hybridation des pratiques, on constate que les évolutions de la médiation culturelle par le numérique construisent dans les cas décrits de nouveaux liens esthétiques entre objets artistiques, patrimoniaux, créateurs, médiateurs, amateurs et publics. Processus de transformation, la médiation est produite par différents acteurs (humains et non humains) et plusieurs auteurs montrent que l'hybridation ne provient pas seulement des techniques ou du mixage de pratiques culturelles, mais est également produite par des acteurs différents qui semblent partager la médiation de manière plus complexe que dans un schéma artiste/médiateur/public, ce sera le troisième point de discussion proposé. 


\section{4. \\ Médiation culturelle, pratiques amateures et recherche : un processus interculturel ?}

Sur la question des acteurs, les recherches ont elles aussi évolué au fil du temps en complexifiant la notion de médiation. Sans souci d'exhaustivité, je ne saisirai ici que deux aspects de cette question, que me suggèrent certains travaux récents mais dont le questionnement se trouve déjà présent dans certains articles plus anciens : la question du rôle des amateurs dans la médiation et celle de la co-construction de la médiation culturelle par les professionnels et les chercheurs.

Bien qu'évoluant dans le temps, la question de la participation collaborative n'est pas tout à fait récente, puisqu'elle est déjà présente dans le projet de la "Nouvelle muséologie » des années 1980, et analysée dans les travaux sur les amateurs (Hennion, 2004b). Plusieurs articles d'EDC ont décrit des pratiques de partenariat de différentes catégories d'acteurs (Van Braekel, 1998) et la manière dont amateurs et publics interviennent dans le processus de médiation du patrimoine urbain (Da-Lage et al., 2008 ; Watremez, 2008) ; plus récemment, E. Lambert, J.-T. Julia et J. Deramond montrent comment des discours de médiation créés par des habitants lors de visites amènent à mieux définir le patrimoine (Lambert et al., 2015). L'article de Lisa Chupin sur la documentation participative des herbiers fait écho à un article d'EDC 36 (Casemajor-Lousteau, 2011) ; les deux montrent l'influence des technologies numériques sur cette évolution du rôle des amateurs et publics dans la médiation culturelle participative. "Le dispositif opère un déplacement par rapport à la communication institutionnelle muséale en constituant un espace de médiation permettant aux profanes de s'approprier les techniques documentaires des collections scientifiques » (Chupin, 2016, 46). Ce qui est en jeu ici est le rôle de la médiation non seulement dans l'interprétation mais dans la constitution de données qui créent de nouveaux patrimoines.

Dans la mesure où la médiation culturelle est un construit, les modalités de cette construction sont importantes et la responsabilité des chercheurs dans la construction de la notion ne peut-être évacuée. Deux articles publiés en 1998 (EDC 21) décrivent l'implication de chercheurs dans la production de médiation soit à partir d'expériences personnelles de médiation : une " observation participante dans le monde semi-professionnel du rock » (Van Braekel, 1998 p. 29) ou en évoquant des négociations difficiles entre ethnologues et maison de disques (Da Lage, 1998, 74-75). Ce lien va s'accentuer au fil des années 2000, la recherche universitaire se structurant davantage autour de projets financés, de recherches-action, dans lesquels le monde de la recherche se retrouve lié au monde professionnel ou au monde associatif (Trouche et Courbières, 2015); de nombreux travaux en SIC sur la médiation décrivent ces projets concrets, orientés vers une visée de développement culturel. Les 
questionnements se construisent donc en lien avec des politiques de médiation, créent des confrontations de point de vue entre professionnels, amateurs, publics et chercheurs.

Ainsi, dans plusieurs articles d'EDC 46, centré sur les musées, on voit que la médiation culturelle liée au développement des dispositifs numériques correspond à un besoin de renouvellement institutionnel qu'accompagnent les chercheurs (Tardy et Renaud, 2016). Le projet d'observation-participante peut devenir, un « espace d'expérimentation sur les solutions conceptuelles, technologiques et organisationnelles des modes d'inscription des données scientifiques dans le web de données ouvert " (Juanals et Minel, 2016, 20) ; comme dans d'autres cas de recherche réunissant chercheurs et institutions, l'article souligne que les enjeux se posent aussi en termes de " position d'autorité scientifique, de visibilité et d'image institutionnelle des établissements » (Juanals et Minel, 2016, 2). Il est intéressant de noter qu'ici, la recherche fait elle-même médiation entre différentes catégories d'acteurs et de conception, d'institutions et qu'elle s'analyse comme une pratique de médiation liée à son terrain. C'est également ce que soulignent dans un autre contexte, Y. Jeanneret et $\mathrm{C}$. Rondo dans le numéro de la revue Les Enjeux de l'information consacré aux médiations, en insistant sur l'obligation de redévelopper une réflexion sur la médiation dans le cadre d'une recherche-action :

«En effet, ce cadre, bien spécifique, amène le chercheur à faire se rencontrer son approche de la médiation, elle-même liée à une théorie de la communication, avec celles qui circulent dans l'institution et qui sont mobilisées, tant par les professionnels de la médiation [...] que par les acteurs de conduite d'un projet défini politiquement » (Jeanneret et Rondo, 2013, 138). La pratique de la recherche se trouve alors en prise avec « le statut multiple de la médiation » (Jeanneret et Rondo, 2013, 138).

Cette même obligation de réflexion sur le sens de la médiation est d'ailleurs soulignée par J. Dalbavie, É. Da Lage et Gellereau (EDC 46) par rapport à la position du chercheur au sein du collectif. Nous montrons comment des expérimentations de recherche construites dans des projets collaboratifs portés par des partenaires professionnels du développement numérique, des chercheurs et des institutions muséales amènent à produire des expériences de médiation et comment les chercheurs négocient avec les producteurs du dispositif de médiation numérique et les institutions un positionnement d'analyse qui refuse d'être assigné à la position d'analyste des usages :

« Dans ce contexte, la recherche apparaît comme un lieu nécessaire de médiation depuis lequel interroger l'intrication toujours plus forte entre dispositifs techniques, expérimentations scientifiques et innovations politiques [...]. Par ailleurs, le travail de terrain permet d'interroger les conceptions, imaginaires techniques partagés par les publics et les concepteurs plutôt que de les naturaliser dans nos méthodes " (Dalbavie et al., 2016, 115). 
Dans d'autres travaux, É. Da Lage et moi-même avons souligné ce rôle de la recherche dans la médiation en décrivant un travail de recherche réalisé avec des amateurs du patrimoine dans lequel « la recherche elle-même devient un dispositif de médiation fondé à la fois sur l'intercompréhension de mondes différents et une transformation par la médiation " (Da Lage et Gellereau, 2015, 68).

Il s'agit donc ici de processus interculturels de médiation dans lesquels non seulement plusieurs acteurs animent des manières différentes d'interpréter les œuvres où le patrimoine, mais construisent et négocient ensemble des méthodes de travail qui aident à comprendre des pratiques différentes de l'art ou du patrimoine et mettent en commun leurs expériences pour produire de nouveaux dispositifs. La dynamique de la médiation ne devient-elle pas alors interculturelle ? Études de communication a enrichi cette réflexion en montrant comment ces médiations collaboratives peuvent être des ressources pour les institutions, mais également participer d'une démocratisation dans l'appropriation des patrimoines. En rendant visibles ces diverses interprétations et transformations par la médiation, ces recherches confirment le caractère dynamique du processus de médiation et le métissage des cultures professionnelles et amateures, l'hybridation des pratiques de médiation. Elles engagent aussi le chercheur dans une position de reconnaissance de la médiation non comme transmission normative mais comme processus ouvert et imprévisible.

\section{Conclusion}

Ces quelques pistes de questionnement, retravaillées à partir des contributions de la revue et du dialogue qu'elles ouvrent avec des recherches récentes, visaient à éclairer le rôle joué par Études de communication dans l'analyse des processus d'évolution de la médiation culturelle et la manière de les construire comme objet de recherche. En reliant trois questions, en discutant d'une vision réductrice de la médiation culturelle comme un "entre-deux ", alors qu'elle est analysée par plusieurs articles de la revue comme processus dynamique de transformation, s'actualisant au fil des développements technologiques dans des pratiques hybrides, processus de rencontre visant à construire du commun partagé, on obtient pour résultat de complexifier une notion toujours en débat. La position exprimée ici vise à montrer l'enrichissement constant de la notion au fil de pratiques ancrées dans les évolutions des mondes de la culture, des pratiques des acteurs de la médiation et des publics. 
Bibliographie

Bonaccorsi J., Croissant V. (2015).

"Votre mémoire culturelle » : entre logistique numérique de la recommandation et médiation patrimoniale. Le cas de Sens Critique ». In Études de communication, $n^{\circ} 45$, p. 129-148.

Bordeaux M.-C. (2007). « La médiation culturelle, un art de l'entre-deux "?. In Les Cahiers de la SFSIC, 1, p. 10-11.

Bordeaux M.-C. (2011). "La médiation culturelle, symptôme ou remède ? Pistes de réflexions pour les arts de la scène ". In La médiation culturelle dans les arts de la scène, Lausanne, La Manufacture, p. 23-36.

Bordeaux M.-C., Caillet E. (2013). "La médiation culturelle : pratiques et enjeux théoriques ". In Culture et Musées Hors-série, p. 139-163.

Caillet E., Lehalle E. (1995). À l'approche du musée, la médiation culturelle, Lyon, Presses Universitaires de Lyon.

Casemajor Loustau N. (2011). « La contribution triviale des amateurs sur le Web : quelle efficacité documentaire? ». In Études de communication, $\mathrm{n}^{\circ} 36$, p. 39-52.

Caune J. (1995). «Pour une politique de la médiation artistique ». In MEI, vol. 3. Disponible sur https://www. mei-info.com/revue/3/media-infor- mation-quest-ce-que-la-culture/ (page consultée le 30 août 2017).

Caune J. (1999). Pour une éthique de la médiation, Grenoble, Presses Universitaires de Grenoble.

Chaumier S., Mairesse F. (2013). La médiation culturelle, Paris, Armand Colin.

Chupin L. (2016). « Documentarisation participative et médiation du patrimoine scientifique numérisé. Le cas des herbiers ». In Études de communication, $n^{\circ} 46$, p. 33-50.

Dalbavie J., Da Lage É., Gellereau M. (2016). « Faire l'expérience de dispositifs numériques de visite et en suivre l'appropriation publique : vers de nouveaux rapports aux œuvres et aux lieux de l'expérience? ». In Études de communication, $n^{\circ} 46$, p. 109-128.

Da Lage É. (1998). « La collection de disques de 'musiques du monde', exemple de pratiques de médiation ". In Études de communication, $\mathrm{n}^{\circ} 21$, p. 67-81.

Da Lage É., Gellereau M. (2015). "Produire de la connaissance avec les amateurs. La recherche comme dispositif de médiation sociale et culturelle ". In Sciences de la société, $n^{\circ}$ 93, p. 57-78.

Davallon J. (2004). « La médiation : la communication en procès ? ». In MEI, 19, p. 37-59. 
Delcambre P. (1998). « Postface.

Penser les pratiques culturelles

en se saisissant du concept

de médiation ». In Études de

communication, $\mathrm{n}^{\circ} 21$, p. 137-143.

Desvallées A., Mairesse F. (dir.) (2011).

Dictionnaire encyclopédique de

muséologie, Paris, Armand Colin.

Gellereau M. (1998a).

"Présentation ». In Études

de communication, Dossier

Médiations culturelles, dispo-

sitifs et pratiques, $\mathrm{n}^{\circ} 21$.

Gellereau M. (1998b). « Dispositif

télévisuel et médiations : une étude

de documentaires et reportages

consacrés aux gens ordinaires ». In

Études de communication, Dossier

Médiations culturelles, dispositifs

et pratiques, $\mathrm{n}^{\circ} 21$, p. 97-108.

Gellereau M. et al. (2000). Médiation des cultures, Lille, Édition du

Conseil scientifique de l'Université

Charles-de-Gaule - Lille 3.

Gellereau M. (2016). « Médiations culturelles et patrimoniales : partager des expériences culturelles pour construire du commun ". In Servais et al., La médiation.

Théories et terrains, De Boeck supérieur, Louvain-la-Neuve.

German R. (2016). « La médiation numérique et l'espace muséal en représentation : les portfolios vidéos d'agences de design prestataires de musées ». In Études de communication, vol. 46, p. 51-70.
Giet S. (1998). « Nous Deux, un dispositif de médiation culturelle? ». In Études de communication, $\mathrm{n}^{\circ} 21$, p. 111-122.

Georgescu Paquin A. (2015).

"Les mappings vidéo sur le patrimoine bâti comme forme d'hybridation entre œuvre et dispositif de médiation ". In Études de communication, $n^{\circ} 45$, p. 53-76.

Hennion A. (1990). «De l'étude des médias à l'analyse de la médiation : esquisse d'une problématique ". In Médiapouvoirs, $\mathrm{n}^{\circ} 20$, p. 39-52.

Hennion A. (2004a). "Une sociologie des attachements. D'une sociologie de la culture à une pragmatique de l'amateur" $"$ In Sociétés, $n^{\circ} 85$, p. 9-24.

Hennion A. (2004b). « Entretien avec Antoine Hennion ». In MEI, n 19 , p. 30-34.

Jeanneret Y., Rondot C. (2013). "Médiation de la médiation au musée du Louvre. Des logiques de recherche au sein d'un projet politique ». In Les Enjeux de l'information et de la communication 2013/1, vol. 14/1, p. 131-147.

Juanals B., Minel J.-L. (2016). "Les stratégies institutionnelles des musées dans le web de données ouvert : la construction d'un espace muséal partagé en question ". In Études de communication, $\mathrm{n}^{\circ} 46$, p. 17-32. 
Lafortune J.-M. et al., (2012). La médiation culturelle. Le sens des mots et l'essence de la pratique, Québec, Presses Universitaires du Québec.

Lambert E., Julia J.-T., Deramond J. (2015). « La ville en live. Itinéraires numériques et artistiques à travers le patrimoine urbain ». In Études de communication, $n^{\circ} 45$, p. 113-128.

Lamizet B. (2000). La médiation culturelle, Paris, L'Harmattan.

Laplantine F., Nouss A. (1997). Le métissage, Paris, Flammarion.

Leclercq E. (1998). "Artistes

humoristiques: du réseau comme médiation ". In Études de communication, $n^{\circ} 2$, p. 13-27.

Le Marec J. (2001). « En guise d'épilogue. Pratiques interprétatives : entre méthodes et sens commun ". In Études de communication, Dossier L'interprétation : entre élucidation et création, $n^{\circ} 24$, p. 125-136.

Le Marec J. (2007). Publics et musées. La confiance éprouvée, Paris, L'Harmattan.

Servais C. (2016). " Qu'est-ce que la médiation ? Examen critique d'une déconstruction de la communication ". In Servais et al., La médiation. Théories et terrains, De Boeck supérieur, Louvain-la-Neuve.

Tardy C., Renaud L. (2015). «Introduction ». In Études de communication, Dossier Pratiques d'espace. Les médiations des patrimoines vers la culture $d u$ numérique? (1), $n^{\circ} 45$, p. 7-14.

Tardy C., Renaud L. (2016). "Introduction ». In Études de communication, Dossier Regards sur l'innovation. Les médiations des patrimoines vers la culture $d u$ numérique? (1), $n^{\circ} 46, \mathrm{p} .7-16$.

Trouche D., Courbières C. et al. (2015). La recherche sur projet en sciences humaines et sociales : lieux, stratégies et contenus. In Sciences de la société, n 93, p. 3-11.

Van Braekel P. (1998). " La figure du manager dans le monde semi professionnel du rock : une pratique de médiation ». In Études de communication, $\mathrm{n}^{\circ} 21$, p. 29-47.

Vandiedonck D. (1998). « Musiques baroque et contemporaine : l'interprétation e(s) t la médiation ". In Études de communication, $\mathrm{n}^{\circ} 21$, p.49-64.

\section{Références Études de communication}

Études de communication, Dossier Médiations culturelles, dispositifs et pratiques, $n^{\circ} 21,1998$.

Études de communication, Dossier L'interprétation : entre élucidation et création, $\mathrm{n}^{\circ}$ 24, 2001.

Études de communication, Dossier Espaces urbains, espaces publics, paroles et interprétations des habitants, $n^{\circ} 31,2008$. 
Études de communication, $n^{\circ} 36,2011$.

Études de communication, Dossier Pratiques d'espace. Les médiations des patrimoines vers la culture du numérique? (1), $n^{\circ}$ 45, 2015.

Études de communication, Dossier Regards sur l'innovation. Les médiations des patrimoines vers la culture du numérique? (1), n 46, 2016. 
